\title{
Adaptação transcultural para uso no Brasil do Instrumento Survey of Technology Use (SOTU Br)
}

\author{
Cross-cultural adaptation for use in Brazil of Survey of Technology Use \\ (SOTU Br)
}

\section{Adaptación transcultural para uso en Brasil del Instrumento Survey of Technology Use (SOTU Br)}

\section{* Ligia Maria Presumido Braccialli}

Professora doutora na Universidade Estadual Paulista Júlio de Mesquita Filho, São Paulo, São Paulo, Brasil. ligia.braccialli@unesp.br - https://orcid.org/0000-0002-2540-3725

\section{** Gabriele Barbaresco Rabadan}

Graduanda pela Universidade Estadual Paulista Júlio de Mesquita Filho, São Paulo, São Paulo, Brasil. gabibarbarescorabadan@hotmail.com

\section{*** Marina Gouvêa Malheiros}

Graduanda pela Universidade Estadual Paulista Júlio de Mesquita Filho, São Paulo, São Paulo, Brasil. marinagmalheiros@hotmail.com

\section{**** Marcia Scherer}

Doutora pela Universidade de Rochester, Rochester, Nova lorque, Estados Unidos. impt97@aol.com - https://orcid.org/0000-0001-8374-6526

Recebido em 01 de outubro de 2018

Aprovado em 11 de janeiro de 2019

Publicado em 05 de junho de 2019

\section{RESUMO}

A taxa de abandono ou não uso de recursos de tecnologia assistiva adquirido é considerada alta, apesar dos esforços dos profissionais no momento da prescrição. A literatura tem apontado que o uso de instrumentos de avaliação padronizados e baseados em modelos conceituais tem contribuído para maior aceitabilidade desses recursos. O estudo teve como objetivo traduzir e adequar culturalmente o instrumento Survey of Technology Use (SOTU) que compõe o modelo conceitual Matching Person and Technology (MPT) para utilização no Brasil (SOTU Br). Trata-se de uma pesquisa do tipo metodológica. Participaram do estudo profissionais especialista em dispensação de recursos de tecnologia assisitiva e pessoas com deficiência que fazem uso de tecnologia assistiva. $O$ estudo foi dividido em cinco etapas: 1) tradução para o português; 2) tradução conciliada; 3) retrotradução; 4) análise de equivalência de itens; 5) pré-teste. Os resultados indicaram que a versão traduzida e adaptada para o português do Brasil teve um alto índice de concordância e uma 
boa aceitabilidade. Conclui-se que a versão em português do SOTU pode ser utilizada por profissionais da educação e da saúde para avaliar a predisposição de uso de tecnologia assistiva e tecnologia de comunicação e informação, por adultos e crianças com deficiência, por meio de suas atitudes e experiências.

Palavras-chave: Tecnologia assistiva; Adaptação transcultural; Educação especial.

\section{ABSTRACT}

The rate of abandonment or non-use of acquired assistive technology resources is considered high, despite the efforts of professionals at the time of prescription. The literature has pointed out that the use of standardized assessment instruments based on conceptual models has contributed to the greater acceptability of these resources. The purpose of the study was to translate and culturally adapt the Survey of Technology Use (SOTU) instrument that makes up the conceptual model of Matching Person and Technology (MPT) for use in Brazil (SOTU Br). This is a methodological research. Participating in the study were specialist practitioners in dispensing assistive technology resources and people with disabilities who make use of assistive technology. The study was divided into five stages: 1) translation into Portuguese; 2) reconciled translation; 3) back-translation; 4) analysis of equivalence of items; 5 ) pre-test. The results indicated that the translated and adapted version of Brazilian Portuguese had a high degree of agreement and good acceptability. It is concluded that the Portuguese version of SOTU can be used by education and health professionals to assess the predisposition to use assistive technology and communication and information technology for adults and children with disabilities through their attitudes and experiences.

Keywords: Assistive technology; Transcultural adaptation; Special education.

\section{RESUMEN}

La tasa de abandono o no uso de recursos de tecnología de apoyo es considera alta, a pesar de los esfuerzos de los profesionales en el momento de la prescripción. La literatura ha señalado que el uso de instrumentos de evaluación estandarizados y basados en modelos conceptuales contribuye a una mayor aceptabilidad de esos recursos. Este estudio tuvo como objetivo traducir y adecuar culturalmente el instrumento Survey of Technology Use (SOTU) que compone el modelo conceptual Matching Person and Technology (MPT) para ser utilizado en el Brasil (SOTU Br). Se trata de una investigación del tipo metodológico. Participaron del estudio especialistas que distribuyen recursos de tecnología de apoyo y personas con discapacidad que hacen uso de esas tecnologías. El estudio se dividió en cinco etapas: 1) traducción al portugués; 2) traducción conciliada; 3) retrotraducción; 4) análisis de equivalencia de los ítems; 5) preprueba. Los resultados indicaron que la versión traducida y adaptada para el portugués de Brasil tuvo un alto índice de concordancia y una buena aceptabilidad. Se concluye que la versión portuguesa del SOTU puede ser utilizado por los profesionales de la educación y la salud para evaluar la 
predisposición de uso de la tecnología de apoyo y la tecnología de comunicación e información para adultos y niños con discapacidad, a través de sus actitudes y experiencias. Palabras clave: Tecnología de apoyo; Adaptación transcultural; Educación Especial.

\section{Introdução}

As questões cruciais para implementação de política de dispensação de tecnologia assistiva (TA) são semelhantes em países com alta, média e baixa renda, tais como: serviços mal estruturados; exclusão do usuário no processo de dispensação; e equipe multiprofissional não treinada e não familiarizada com a política de dispensação do país. Em países de baixa e média renda, soma-se a esses fatores a inadequada oferta de produtos de TA (MACLACHLAN et al., 2018).

O termo "dispensação de tecnologia assistiva" envolve tudo o que é necessário para garantir que uma pessoa com deficiência, que possa se beneficiar da TA, obtenha a solução mais apropriada para ela. Para uma dispensação de TA ideal, no entanto, alguns elementos são essenciais: 1) que a prestação de serviços, que é o processo pelo qual um indivíduo vai obter uma solução de TA, atenda às necessidades do usuário; 2) boa qualidade e preços acessíveis dos produtos; 3) as pessoas envolvidas, usuários finais e profissionais, conheçam as soluções existentes; 4) haver serviços profissionais para fornecer aconselhamento e apoio; 5) políticas e procedimentos para decidir sobre a elegibilidade para certas soluções e mecanismos de financiamento; 6) treinamento em uso; 7) serviços de acompanhamento; 8) infraestrutura para manutenção e reparos. Todos esses elementos podem ser organizados de maneiras diferentes, mas precisam estar em vigor para que a dispensação seja efetiva (DE WITTE et al., 2018).

Sabe-se que os produtos de tecnologia assistiva (TA) funcionam melhor quando correspondem às necessidades e aos objetivos do indivíduo e ao ambiente no qual as atividades são ou serão realizadas, e torna-se crucial o envolvimento da pessoa com deficiência de forma direta e abrangente em todas as fases do ciclo de vida do produto, desde a geração de ideias até a medição dos resultados (SMITH et al., 2018).

Nessa perspectiva, dois modelos conceituais de dispensação de TA se destacam na literatura: o Matching, Person \& Technology (MPT) e o modelo Assistive Technology Assessment Process (ATA). 
O modelo Processo de Avaliação de Tecnologia Assistiva propõe um conjunto sequencial de avaliações para adequar uma pessoa à tecnologia assistiva (TA) com a ajuda de profissionais de diferentes áreas de conhecimento e com experiência em prestação de serviços especializados em tecnologia de reabilitação (FEDERICI et al., 2015). O modelo de processo ATA baseia-se em alguns pilares: (a) fundamenta-se na CIF, sendo que todas as dimensões propostas pela CIF que interferem na funcionalidade do usuário são consideradas durante todo o processo de solicitação da TA pelo usuário, durante a escolha da solução de TA mais apropriada, e durante o período de treinamento e acompanhamento de uso; (b) incorpora os conceitos e utiliza os instrumentos de avaliação proposto pelo modelo MPT; (c) segue as recomendações propostas pela Association for the Advancement of Assistive Technology in Europe (AAATE) que preconiza que a superação de uma incapacidade vai além da disponibilização de um dispositivo, deve haver a adequação do dispositivo de TA às necessidades do usuário, e que essa adequação pode depender do ambiente em que será utilizada; e (d) a necessidade de uma equipe multiprofissional com formação em recursos humanos e em TA, que tem como função analisar e avaliar a interação entre o usuário e a tecnologia (FEDERICI; BORSCI, 2016).

O MPT foi desenvolvido por Scherer (SCHERER; CRADDOCK, 2002) e tem sido considerado o conceito mais conhecido e utilizado durante a seleção de produtos de TA (ALVES; MATSUKURA; SCHERER, 2017; BRACCIALLI, 2017; SCHERER, 2017). Esse modelo conceitual considera que o uso apropriado de produtos de TA deve ser uma atividade pessoal, e sensível ao contexto; com a devida consideração pelo ambiente social e físico. O MPT, destaca que um único recurso e com um único tamanho não serve para todos os usuários, atividades e situações. Assim, a combinação de um produto de tecnologia assistiva com o usuário deve incluir um processo de avaliação detalhada sobre as necessidades e predisposição de uso do dispositivo (SMITH et al., 2018). Trata-se de uma abordagem colaborativa em que o usuário de TA e o profissional, especialista no trabalho com pessoas com deficiência, trabalham em conjunto, a fim de selecionar a tecnologia mais apropriada para o usuário.

O MPT propõe que a predisposição de uso de uma tecnologia por um indivíduo deve ser analisada em relação a três aspectos: a) os fatores psicossociais; b) o ambiente no qual o usuário interage com o recurso; e c) fatores específicos do recurso de tecnologia (SCHERER et al., 2005; ALVES; MATSUKURA; SCHERER, 2017). A avaliação ocorre, 
tendo como base uma abordagem individualizada e centrada no cliente e tem como objetivo realizar uma combinação adequada do usuário com a tecnologia, por meio dos instrumentos: Survey of Technology Use - SOTU, Assistive Technology Device Presdiposition Assesssment - TD-PA, Educational Technology Device Predisposition Assessment - ET PA, Workplace Technology Device Predisposition Assessment - WT PA, Healthcare Technology Device Predisposition Assessment - HCT PA (SCHERER; FEDERICl, 2015). Encontram-se disponíveis em língua portuguesa os instrumentos Assistive Technology Device Presdiposition Assesssment - ATD-PA (ALVES; MATSUKURA; SCHERER, 2017) e o Educational Technology Device Predisposition Assessment - ET PA (BRACCIALLI, 2017).

Dentre os instrumentos que compõem o modelo MPT, O SOTU tem sido utilizado em diferentes culturas para medir a atitude do usuário em relação à tecnologia (SCHERER; GLUECKAUF, 2005). Na literatura, existem diferentes pesquisas que utilizaram o SOTU para obter informações, de usuários adultos (FEDERICl et al., 2003; ARBERAS, 2017), idosos (VRKLJAN, 2010; VROMAN; ARTHANAT; LYSACK, 2015; ARTHANAT; VROMAN; LYSACK, 2016) e crianças (FEDERICl et al., 2015) com deficiência, em relação a predisposição de uso de tecnologia assistiva e tecnologia de comunicação e informação por meio de suas atitudes e experiências (SCHERER; CRADDOCK, 2002).

O SOTU destaca-se como um instrumento com boa validade concorrente e discriminante (FEDERICl et al., 2003), assim o estudo teve como objetivo traduzir e adequar culturalmente o instrumento Survey of Technology Use (SOTU) que compõe o modelo conceitual Matching Person and Technology (MPT) para utilização no Brasil (SOTU Br).

\section{Método}

Foi solicitada e autorizada a realização da tradução e adaptação cultural para a língua portuguesa do instrumento Survey of Technology Use (SOTU). O projeto foi encaminhado e aprovado pelo Comitê de Ética em Pesquisa da Faculdade de Filosofia e Ciências da Universidade Estadual Paulista (Unesp) com parecer número 1399-306 e CAAE 52363815300005406 . 


\section{Delineamento de Pesquisa}

No Quadro 1 foram introduzidas as características dos participantes para cada etapa da pesquisa. Todos os participantes assinaram o Termo de Consentimento Livre e Esclarecido.

Figura 1 - Etapas do estudo e características dos participantes

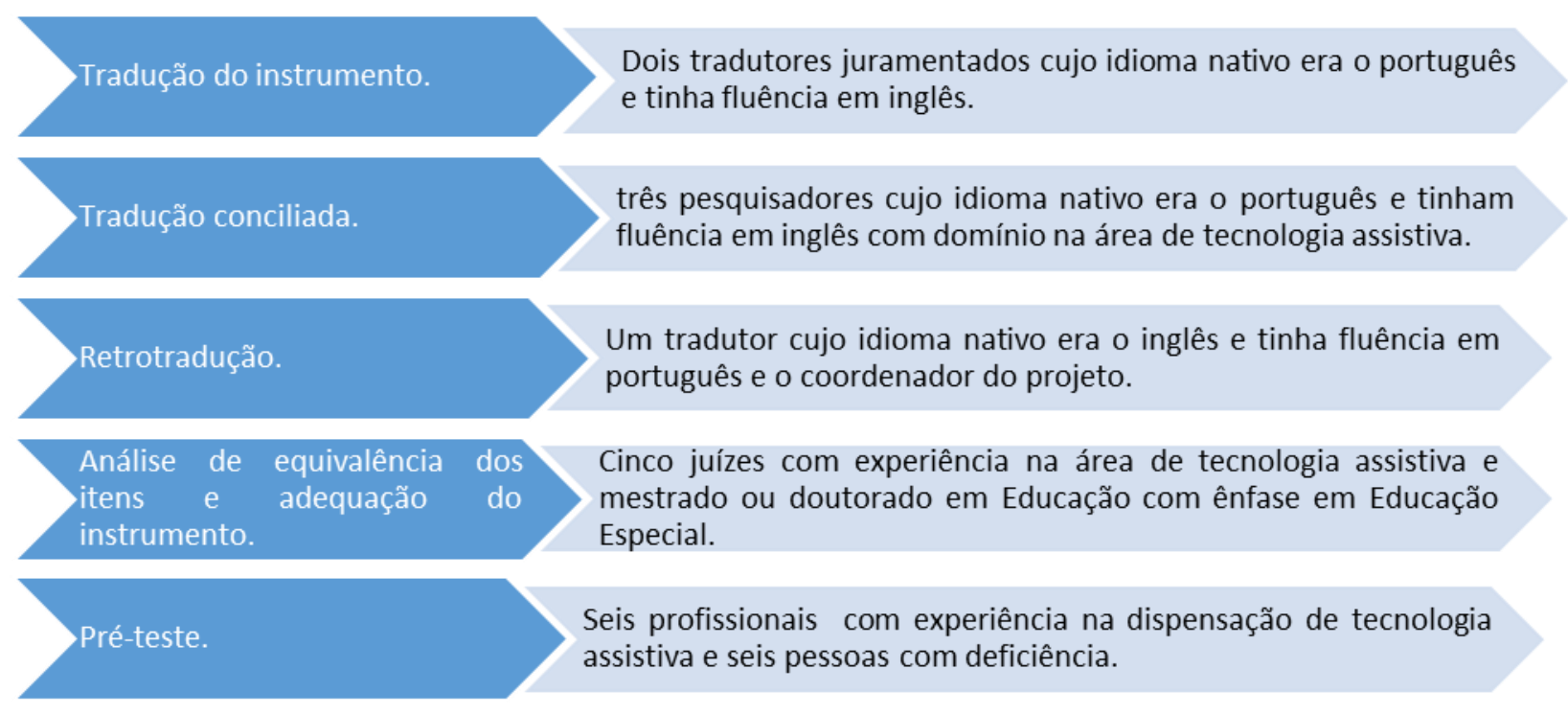

Fonte: os autores.

O instrumento SOTU é um questionário para realização de pesquisas do tipo survey, utilizado com objetivo de avaliar a predisposição geral da pessoa em usar tecnologia. Survey é um tipo de instrumento utilizado para coletar informações de pessoas sobre suas ideias, sentimentos, planos, crenças, origem social, educacional e financeira (PASQUALLI, 1996).

O questionário SOTU é composto por duas versões, a versão respondida pelo profissional que irá prescrever a tecnologia e a versão respondida pelo próprio usuário. $\mathrm{O}$ usuário deve preencher o formulário SOTU consumidor (SOTU-C), que é composto por uma lista de itens, que investiga as suas experiências e os seus sentimentos em relação as tecnologias utilizadas no momento, são listadas todas as tecnologias utilizadas e o sentimento em relação ao conforto de uso. A versão profissional SOTU profissional (SOTUP) é idêntica à versão do consumidor, no entanto são informações obtidas sob óptica do profissional. As duas formas foram concebidas para serem usadas em conjunto, de modo 
que as diferentes perspectivas, do consumidor e do profissional, tornem-se evidentes e, assim, possam ser resolvidas (SCHERER, 2008).

O SOTU examina os seguintes aspectos que influenciam o uso ou não uso de dispositivos de tecnologia pelo consumidor: (1) tecnologias frequentemente usadas pelo consumidor; (2) experiências do consumidor com as tecnologias atuais; (3) perspectivas do consumidor sobre novas tecnologias; (4) atividades típicas do consumidor; (5) características pessoais/ sociais do consumidor. Todos os itens são apresentados em um formato semântico de três pontos para que o consumidor possa expressar os seus sentimentos em relação a essas influências (positivo, neutro, negativo). O SOTU-C, pode ser auto preenchido ou pode ser utilizado em formato de entrevista. Após o preenchimento do SOTU-C o profissional deve preencher o SOTU-P. O SOTU-C e o SOTU-P são analisados exatamente da mesma maneira. Deve-se totalizar o número de itens positivos, neutros e negativos para cada domínio, em seguida, na seção de pontuação que se encontra no final do questionário, é preenchido os respectivos valores. Por fim, totalize as três colunas (positivo, neutro, negativo) na seção de pontuação (SCHERER, 2008).

O SOTU pode ser usado por qualquer profissional que faça parte da equipe para prescrição de recursos de tecnologia. A equipe deve ter um perfil multidisciplinar, com profissionais com diferentes formação.

\section{Desenvolvimento do estudo}

O processo de tradução e adaptação cultural seguiu recomendações internacionais (GUILLEMIN; BOMBARDIER; BEATON, 1993; BEATON et al., 2000; MELCHIORS; CORRER; FERNÁNDEZ-LLIMOS, 2007; COSTER; MANCINI, 2015; BRACCIALLI et al., 2016). Conforme as recomendações desses autores o estudo foi dividido em cinco etapas: 1) tradução para o português; 2) tradução conciliada; 3) retrotradução; 4) análise de equivalência de itens; e 5) pré-teste.

\section{Primeira Etapa: Tradução para o português}

Inicialmente as duas versões do instrumento original foram traduzidas para o idioma português do Brasil por dois tradutores juramentados. Foram realizadas duas traduções independentes por dois tradutores cuja língua nativa era o português do Brasil e tinham fluência em inglês, língua em que o instrumento original foi desenvolvido. $O$ coordenador do projeto tinha experiência na área de tecnologia assistiva, e com instrumentos de avaliação, além de ter domínio do idioma inglês. O coordenador do projeto teve como 
função realizar a mediação com os tradutores de forma independente a fim de resolver dúvidas específicas em relação a terminologia da área de tecnologia assistiva, mas não realizou a tradução dos instrumentos.

Recomenda-se que a tradução inicial seja feita por pelo menos duas pessoas que trabalhem de forma independente, sendo importante que os dois tradutores ou equipes não se comuniquem durante o processo para que possam ser comparadas as versões posteriormente (COSTER; MANCINI, 2015) e para que se tenha mais opções para definir os termos a serem utilizados na versão a ser posta em teste (REICHENHEIM; MORAES, 2007).

Outras recomendações encontradas na literatura para o processo de tradução inicial: (a) deve haver um coordenador com fluência no idioma estrangeiro durante o desenvolvimento da tradução independente (COSTER; MANCINI, 2015); (b) o idioma nativo do tradutor deve ser o idioma para o qual o instrumento foi traduzido (BEATON et al., 2000), sendo importante também que o tradutor tenha familiaridade com o contexto para o qual o instrumento foi desenvolvido assim como com o próprio instrumento já que determinados termos são facilmente compreendidos por profissionais da área, mas não tão claros para pessoas de fora do campo (COSTER; MANCINI, 2015; BRACCIALLI, 2017).

\section{Segunda Etapa: Tradução conciliada}

Nessa etapa foi realizada a comparação das duas versões traduzidas para o português. Três pesquisadores cujo idioma nativo era o português, com fluência em inglês e com domínio na área de tecnologia assistiva realizaram em conjunto a leitura de item a item do instrumento, com objetivo de verificar os pontos em que havia diferenças, decidir qual a melhor tradução, sugerir nova tradução caso necessário. Ao final dessa etapa originou-se a versão um do instrumento em língua portuguesa.

A análise dessa etapa foi realizada por meio da frequência absoluta e relativa em relação a opção selecionada pela equipe para cada item do instrumento: tradução realizada pelo tradutor 1, tradução realizada pelo tradutor 2 , traduções iguais e sugestão de nova tradução.

\section{Terceira Etapa: Retrotradução}

Nesta etapa, a versão um do instrumento em língua portuguesa foi retraduzida para o seu idioma original, o inglês, e comparada à versão original. A retrotradução, nesse estudo, 
foi realizada por um tradutor que tinha como língua nativa o inglês e era fluente em português e as dificuldades foram discutidas e resolvidas com o coordenador do estudo.

Ao finalizar a retrotradução foi feito o encaminhamento dessa versão, por meio de email, ao autor do instrumento. Este analisou a versão e retornou para o pesquisador com suas considerações. Após a análise pelo pesquisador e ajustes nos itens sugeridos pelo autor teve origem a versão dois do instrumento.

A retrotradução ou tradução-reversa é um tipo de verificação de validade por destacar inconsistências grosseiras ou erros conceituais de tradução (BEATON et al., 2000). O tradutor deve ter o inglês como primeiro idioma (COSTER; MANCINI, 2015).

Para resolver questões maiores, o coordenador do processo de tradução e os tradutores-reversos podem discutir as diferenças e, a partir das discussões, chegar a um acordo sobre a melhor tradução (REICHENHEIM; MORAES, 2007).

$\mathrm{O}$ instrumento retrotraduzido deve ser encaminhado ao autor e/ou editor original do instrumento para que faça uma revisão, afim de garantir que a tradução esteja precisa e que a mesma mantenha as características mais importantes do instrumento original (COSTER; MANCINI, 2015). Busca-se ao final dessa etapa a colaboração direta do autor com a equipe de tradução, assim, caso o autor encontre termos que não sejam idênticos aos da versão original, ele pode discutir com a equipe para determinar se o termo traduzido mantém o sentido pretendido (COSTER; MANCINI, 2015).

\section{Quarta Etapa: Análise de equivalência dos itens e adequação do instrumento}

Tem sido recomendada a revisão das seguintes equivalências:

1) equivalência conceitual e de itens - para que sejam obtidas tais equivalências, é necessária uma discussão com um grupo de especialistas que possam avaliar a populaçãoalvo em que o instrumento será utilizado. Isto tem como finalidade explorar se os diferentes domínios abarcados pelo instrumento original na definição dos conceitos de interesse seriam relevantes e pertinentes ao novo contexto para qual foi adaptado. No processo, avalia-se a pertinência dos itens para a captação de cada um desses domínios.

2) equivalência semântica - a análise da equivalência semântica consiste na capacidade de transferência de sentido dos conceitos presentes no instrumento original para a versão que está sendo desenvolvida.

3) equivalência operacional - refere-se a uma comparação entre os aspectos de utilização de um instrumento nas populações-alvo e fonte, de modo que a eficácia seja 
semelhante mesmo que os modus operandi não sejam os mesmos (REICHENHEIM; MORAES, 2007).

Com base nessas premissas, nessa etapa do estudo, foi realizada a avaliação da equivalência entre a versão dois do instrumento e o instrumento original por uma equipe composta por cinco juízes com experiência na área de tecnologia assistiva e com mestrado ou doutorado em Educação com ênfase em Educação Especial.

Para a avaliação de equivalência foi encaminhado aos juízes um formulário construído especificamente para esse fim e denominado "Protocolo de avaliação de equivalência". O protocolo seguiu as recomendações sugeridas por Braccialli (2017).

Os juízes avaliaram o instrumento sob os seguintes aspectos: (1) equivalência semântica; (2) equivalência experiencial; (3) equivalência conceitual; (4) equivalência operacional. Foram orientados a preencher seus dados de identificação no Protocolo de avaliação de equivalência; a ler atentamente a versão do instrumento traduzido para o português, item a item; imediatamente após a leitura de um item do instrumento responder no Protocolo a alternativa que considerava mais adequada em relação a equivalência semântica, experiencial, conceitual e operacional e caso não concordasse com o item era solicitada sugestão par modificação.

Após a devolução dos protocolos, preenchidos pelos juízes, foi realizada a análise por meio do índice de concordância, como proposto por Fagundes (1999), por meio da fórmula:

$$
\text { índice de concordância }=\frac{\text { concordâncias }}{\text { concordância }+ \text { discordâncias }} \times 100
$$

Valores de Índice de Concordância maiores que 90\% tem uma fidedignidade muito alta; entre $80 \%$ e $89 \%$ consideradas alta; entre $66 \%$ e $79 \%$ é aceitável e valores inferiores a $66 \%$ indicam que as questões são difíceis de compreender (BAUER; GASKELL, 2002). Quando os valores forem inferiores a $66 \%$ recomenda-se que os itens sejam revisados e substituídos por outros com o mesmo conceito. Nessa situação deve ser utilizada as sugestões dos próprios juízes (BRACCIALLI, 2017).

\section{Quinta Etapa: Pré-teste}

Esse processo compreende a aplicação do instrumento traduzido em sujeitos alvos para analisar se os itens foram compreendidos como esperado, pode também, ser usado um grupo de profissionais que não estiveram envolvidos no processo de tradução 
(COSTER; MANCINI, 2015). Para essa etapa, pesquisadores têm recomendado utilizar uma amostra pequena, entre cinco e dez pessoas, pois permite recrutar participantes com perfil específico e altamente crítico que favorecerá a qualidade da análise de itens (FERREIRA et al, 2014).

Deve-se notar que, embora esse estágio forneça informações úteis sobre como a pessoa interpreta os itens do questionário, ele não aborda a validade de construto, a confiabilidade ou padrões de resposta do item que também são críticos para descrever uma adaptação transcultural bem-sucedida (BEATON et al., 2000). Quanto maior as diferenças entre o idioma e a cultura, entre os países de origem e de tradução do instrumento, mais crítica a avaliação deve ser (COSTER; MANCINI, 2015).O objetivo dessa etapa foi verificar quais partes do instrumento podiam não estar com sentido claro ou termos que soassem estranhos/não familiares.

A versão profissional foi respondida, no formato autopreenchimento, por seis profissionais com experiência em dispensação de tecnologia assistiva. Cada profissional que participou do pré-teste, realizou uma entrevista com uma pessoa com deficiência para preenchimento da versão consumidor. Nessa etapa os participantes respondiam o questionário e verificavam, item a item, se havia compreensão do conteúdo e caso surgisse alguma dúvida era feita a sugestão de modificação. Após a coleta de dados foi realizada a análise por meio do índice de concordância.

\section{Resultados}

Os resultados foram apresentados em tópicos correspondentes a cada etapa do estudo: 1) análise da tradução conciliada; 2) análise da retrotradução; 3) análise de equivalência dos itens; e 4) pré-teste.

\section{Análise da tradução conciliada}

$\mathrm{Na}$ Tabela 1 foi apresentada a análise de frequência relativa e absoluta em relação a opção de tradução dos itens pelos participantes do estudo para elaboração da primeira versão do instrumento na língua portuguesa.

Tabela 1 - Frequência absoluta e relativa em relação a opção de tradução

\begin{tabular}{lcc}
\hline & SOTU - Consumidor & SOTU - Profissional \\
& N (\%) & N (\%) \\
\hline Traduções iguais & $33(37)$ & $41(44)$ \\
Tradutor 1 & $35(39)$ & $34(36)$
\end{tabular}




\begin{tabular}{lll} 
Tradutor 2 & $19(22)$ & $11(12)$ \\
Nova tradução & $02(02)$ & $08(08)$ \\
\hline Total & $89(100)$ & $94(100)$
\end{tabular}

Fonte: os autores.

\section{Análise da Retrotradução}

A versão 1 do instrumento foi retrotraduzida e encaminhada por e-mail para a autora do instrumento original.

A autora fez a análise da retrotradução e retornou suas considerações para a pesquisadora. Em sua análise ela não apontou nenhum termo inadequado ou problema de conteúdo. Dessa forma, a versão dois do instrumento manteve-se igual a versão um.

\section{Análise de equivalência dos itens}

A análise de equivalência de itens foi apresentada separadamente para versão consumidor e versão profissional.

\section{Versão consumidor}

$\mathrm{Na}$ análise de equivalência conceitual nove itens tiveram $75 \%$ de concordância entre juízes e 80 itens tiveram $100 \%$ de concordância, portanto não houve necessidade de modificação de nenhum item. Em relação a análise realizada da equivalência experiencial seis itens tiveram $75 \%$ de concordância entre juízes e 83 itens tiveram $100 \%$ de concordância, não sendo preciso modificar nenhum item. $A$ análise da equivalência operacional indicou que os 89 itens tiveram $100 \%$ de concordância. $\mathrm{Na}$ análise de equivalência semântica um item teve $50 \%$ de concordância, 30 itens tiveram $75 \%$ e 51 itens tiveram $100 \%$ de concordância.

No Quadro 1 foram dispostas as informações referente ao item que teve índice de concordância entre os juízes de $51 \%$ e necessitou de revisão para a versão 3 do instrumento em língua portuguesa para o pré-teste.

Quadro 1 - Equivalência semântica da versão inglês/português do item que necessitou de revisão

\begin{tabular}{|c|c|c|}
\hline Versão original & Versão 1 português & Sugestões \\
\hline $\begin{array}{l}\text { List the technologies that you } \\
\text { use most frequently (for } \\
\text { example, personal computer, } \\
\text { VCR, bank ATM, CD player, } \\
\text { etc.) }\end{array}$ & $\begin{array}{lr}\text { Liste as tecnologias que você } \\
\text { usa com mais frequência (por } \\
\text { exemplo: r computador, } \\
\text { videocasseter } \\
\text { eletrônico, leitor de CD etc.) }\end{array}$ & $\begin{array}{l}\text { Liste as tecnologias que você } \\
\text { usa frequentemente (por } \\
\text { exemplo: computador, } \\
\text { videocassete, caixa } \\
\text { eletrônico, leitor de CD etc.) }\end{array}$ \\
\hline & & $\begin{array}{l}\text { Liste as tecnologias que você } \\
\text { usa mais frequentemente } \\
\text { (por exemplo: computador, }\end{array}$ \\
\hline
\end{tabular}




\begin{tabular}{|l|l|l|}
\hline & $\begin{array}{l}\text { videocassete, caixa } \\
\text { eletrônico, leitor de CD e DVD } \\
\text { etc.) }\end{array}$ \\
\hline
\end{tabular}

Fonte: os autores.

\section{Versão profissional}

$\mathrm{Na}$ análise de equivalência conceitual da versão profissional 78 itens tiveram 100\% de concordância entre juízes, 15 itens tiveram $75 \%$ de concordância e um item teve 50\% de concordância, sendo necessário modificá-lo (quadro 2). Em relação a análise realizada da equivalência experiencial 89 itens tiveram 100\% de concordância entre juízes e cinco itens tiveram $75 \%$ de concordância, não sendo preciso modificar nenhum item. A análise da equivalência operacional indicou que os 94 itens tiveram $100 \%$ de concordância. $\mathrm{Na}$ análise de equivalência semântica 69 itens tiveram 100\% de concordância, 25 itens tiveram $75 \%$, portanto não foi preciso modificá-los.

Quadro 2 - Equivalência conceitual da versão inglês/português do item que necessita de revisão

\begin{tabular}{|l|l|l|}
\hline Versão original & Versão 1 português & Sugestões \\
\hline Poor sense of well-being & $\begin{array}{l}\text { Não sente uma sensação de } \\
\text { bem-estar }\end{array}$ & $\begin{array}{l}\text { Pouca sensação de bem- } \\
\text { estar (não sente é nula, neste } \\
\text { caso a sensação está } \\
\text { diminuída) }\end{array}$ \\
\hline
\end{tabular}

Fonte: os autores.

\section{Pré-teste}

Os dados referentes ao pré-teste, também, foram apresentados separadamente para a versão consumidor e profissional.

\section{Versão consumidor}

A análise do índice de concordância entre os participantes realizados no pré-teste indicou uma taxa alta de concordância, sendo que, todos os itens tiveram valores superiores a $66 \%$, indicando um questionário de fácil compreensão.

Apesar do alto índice de concordância dois juízes deram sugestões em alguns itens específicos (Quadro 3), os quais foram considerados na versão final do instrumento.

Quadro 3 - Sugestões de modificações no instrumento.

\begin{tabular}{|l|l|}
\hline \multicolumn{1}{|c|}{ Itens } & \multicolumn{1}{c|}{ Sugestões } \\
\hline "Pesquisa sobre as tecnologias" & $\begin{array}{l}\text { Foi realizada sugestão para a inclusão de um item } \\
\text { sobre experiências com tecnologia na reabilitação }\end{array}$ \\
\hline $\begin{array}{l}\text { "Experiências gerais com as as } \\
\text { tecnologias utilizadas atualmente" }\end{array}$ & $\begin{array}{l}\text { Foi relatada dificuldade na compreensão das palavras: } \\
\text { criatividade, motivadoras e autoestima }\end{array}$ \\
\hline
\end{tabular}




\begin{tabular}{|l|l|}
\hline $\begin{array}{l}\text { "Algumas de suas características } \\
\text { pessoais/sociais }\end{array}$ & $\begin{array}{l}\text { Foi relatada dificuldade do usuário na compreensão } \\
\text { das palavras: tolerante, otimista, motivado, } \\
\text { perseverante. }\end{array}$ \\
\hline
\end{tabular}

Fonte: os autores.

\section{Versão profissional}

Após a realização do pré-teste, foi feita a análise de concordância entre os profissionais, a qual obteve um resultado de 100\% de concordância. Houve apenas uma observação que relatava a dificuldade do profissional em responder as questões sobre experiências anteriores do usuário com tecnologia.

\section{Discussão}

A tradução e adaptação de um instrumento padronizado para uma outra cultura envolve um processo complexo e sistemático e deve seguir etapas preestabelecidas internacionalmente: tradução para o português, tradução conciliada, retrotradução, análise de equivalência de itens, pré-teste (GUILLEMIN; BOMBARDIER; BEATON, 1993; BEATON et al., 2000; MELCHIORS; CORRER; FERNÁNDEZ-LLIMOS, 2007; COSTER; MANCINI, 2015; BRACCIALLI et al., 2016). Os dados desse estudo indicaram que todas as etapas previstas nesse tipo de pesquisa foram cumpridas integralmente.

Observa-se que na etapa de retrotradução não foram feitas considerações de modificações de termos pela autora do instrumento original. Esses dados indicam que a tradução de um instrumento para um outro idioma pode ser mais adequado e com maior sucesso se alguns pontos forem considerados durante o processo: (a) a seleção de um instrumento relevante e apropriado para a população e para o contexto onde será utilizado; (b) trabalho conjunto entre autores do instrumento e pesquisadores que trabalham no processo de tradução; (c) pesquisador com conhecimento específico na área de conhecimento e com domínio na língua do instrumento original (COSTER; MANCINI, 2015; BRACCIALLI, 2017).

A análise de equivalência de itens teve um alto índice de concordância, tanto na versão do consumidor, quanto na versão do profissional. No entanto, observou-se que na versão do consumidor do instrumento SOTU-C um maior número de sugestões foi realizado. Outros estudos têm indicado uma maior dificuldade na tradução de instrumentos em que o respondente é a pessoa com deficiência, principalmente crianças, quando comparado com instrumentos respondidos por profissionais ou cuidadores (BRACCIALLI et al., 2013; BRACCIALLI, 2017). 
Em relação ao pré-teste somente para a versão SOTU - C os participantes fizeram sugestões de modificações de itens, principalmente devido à dificuldade de a pessoa com deficiência compreender alguns termos. Além dessas dificuldades, foi feita a sugestão de incluir um item sobre experiências com tecnologia em reabilitação. Em relação a essa sugestão, deve-se considerar que o SOTU - C faz parte de um conjunto de instrumentos que compõem o modelo conceitual MPT e que devem ser usados concomitantes, e especificamente os instrumentos Assistive Technology Device Predisposition Assessment (ATD PA) e o Healthcare Technology Device Predisposition Assessment (HCT PA) abordam questões referentes a tecnologia em reabilitação e saúde (ALVES; MATSUKURA, 2014; ALVES; MATSUKURA; SCHERER, 2017; BRACCIALLI, 2017).

Novamente, observou-se que para versão em que o respondente é o profissional, SOTU - P, não houve dificuldades de compreensão, talvez esse processo tenha sido facilitado, pois tanto os juízes e participantes do pré-teste eram especialistas na área de TA e dispensação de recursos.

\section{Conclusão}

A tradução e adaptação transcultural do instrumento Survey of Technology Use (SOTU) para uso no Brasil (SOTU Br) mostrou-se adequada e de fácil compreensão pelos profissionais que prescrevem e pelos usuários de TA.

Considera-se importante a elaboração de um manual em língua portuguesa com informações referentes: (a) população a que destina o instrumento; (b) forma de utilização do instrumento; (c) preenchimento e pontuação do instrumento; (d) profissionais habilitados a utilizar o instrumento.

\section{Referências}

ALVES, A. C. de J.; MATSUKURA, T. S. Revisão sobre avaliações para indicação de dispositivos de tecnologia assistiva. Revista Terapia Ocupacional Universidade de São Paulo, v. 25, n. 2, p. 199-207, 2014.

ALVES, A. C. de J.; MATSUKURA, T. S.; SCHERER, M. J. Cross-cultural adaptation of the assistive technology device-Predisposition assessment (ATD PA) for use in Brazil (ATD PA Br). Disability and Rehabilitation: Assistive Technology, v. 12, n. 2, p. 160-164, 2017.

ARBERAS, E. J. Experiencias y perspectivas tecnológicas en personas con discapacidad auditiva / personas sordas. Revista Chilena de Terapia Ocupacional, v. 17, n. 2, p. 4758, 2017. 
ARTHANAT, S.; VROMAN, K. G.; LYSACK, C. A home-based individualized information communication technology training program for older adults: A demonstration of effectiveness and value. Disability and Rehabilitation: Assistive Technology, v. 11, n. 4, p. 316-324, 2016.

BAUER, M. W.; GASKELL, G. Pesquisa qualitativa com texto: imagem e som: um manual pratico. Rio de Janeiro: Vozes, 2002.

BEATON, D. E. et al. Guidelines for the process of cross-cultural adaptation of self-report measures. Spine, v. 25, n. 24, p. 3186-3191, 2000.

BRACCIALLI, A. C. Tradução e adaptação transcultural do instrumento educational technology predisposition assessment - ET PA. 2017.102 f. Dissertação (Mestrado em Educação) - Faculdade de Filosofia e Ciências, Universidade Estadual Paulista Júlio de Mesquita Filho, 2017.

BRACCIALLI, L. M. P. et al. Translation and validation of the Brazilian version of the Cerebral Palsy Quality of Life Questionnaire for Children - Child report. Jornal de Pediatria, v. 92, n. 2, p. 143-148, 2016.

BRACCIALLI, L. M. P. et al. Quality of life questionnaire for children with cerebral palsy ( CP QOL-CHILD ): translation and cultural adaptation for brazilian portuguese language. Journal of Human Growth and Development, v. 23, n. 2, p. 1-10, 2013.

COSTER, W. J.; MANCINI, M. C. Recommendations for translation and cross-cultural adaptation of instruments for occupational therapy research and practice. Revista de Terapia Ocupacional da Universidade de São Paulo, v. 26, n. 1, p. 50, 2015.

DE WITTE, L. et al. Assistive technology provision: towards an international framework for assuring availability and accessibility of affordable high-quality assistive technology. Disability and Rehabilitation: Assistive Technology, v. 13, n. 5, p. 467-472, 2018.

DEMO, P. Metodologia Científica em Ciências Sociais. São Paulo: Atlas, 1995.

FAGUNDES, A. J. F. M. Descrição, definição, e registro de comportamento. São Paulo: Edicon, 12 ed., 1999.

FEDERICI, S.; BORSCI, S. Providing assistive technology in Italy: The perceived delivery process quality as affecting abandonment. Disability and Rehabilitation: Assistive Technology, v. 11, n. 1, p. 22-31, 2016.

FEDERICI, S. et al. Successful assistive technology service delivery outcomes from applying a person-centered systematic assessment process: a case study. Life Span and Disability XVIII, v. 1, p. 41-74, 2015.

FEDERICI, S. et al. A cross-cultural analysis of relationships between disability selfevaluation and individual predisposition to use assistive technology. Assistive technology: shaping the future., p. 941-946, 2003. 
FERREIRA, L. et al. Guia da AAOS/IWH: sugestões para adaptação transcultural de escalas. Avaliação Psicológica, v. 13, n. 3, p. 457-461, 2014.

GUILLEMIN, F.; BOMBARDIER, C.; BEATON, D. Cross-cultural adaptation of healthrelated quality of life measures: Literature review and proposed guidelines. Journal of Clinical Epidemiology, v. 46, n. 12, p. 1417-1432, 1993.

LIMA, D. V. M. Research design: a contribution to the author. Online Brazilian Journal of Nursing, v. 10, n. 2, 2011.

MACLACHLAN, M. et al. Assistive technology policy: a position paper from the first global research, innovation, and education on assistive technology (GREAT) summit. Disability and Rehabilitation: Assistive Technology, v. 13, n. 5, p. 454-466, 2018.

MELCHIORS, A. C.; CORRER, C. J.; FERNÁNDEZ-LLIMOS, F. Tradução e Validação para o Português do Medication Regimen Complexity Index. Arquivos Brasileiros de Cardiologia, v. 89, n. 4, p. 210-218, 2007.

PASQUALI, L.. Teoria e métodos de medida em ciências do comportamento. Brasília: Universidade de Brasília,1996.

REICHENHEIM, M. E.; MORAES, C. L. Operacionalização de adaptação transcultural de instrumentos de aferição usados em epidemiologia. Revista de Saude Publica, v. 41, n. 4, p. 665-673, 2007.

SCHERER, M. J. The Institute For Matching Person \& Technology: model and assessment process. New York: The Institute for Matching Person \& Technology, Inc., 2008.

SCHERER, M. J. Technology adoption, acceptance, satisfaction and benefit: integrating various assistive technology outcomes. Disability and Rehabilitation: Assistive Technology, v. 12, n. 1, p. 1-2, 2017.

SCHERER, M. J.; CRADDOCK, G. Matching person \& technology (MPT) assessment process. Technology \& Disability, v. 14, p. 125-131, 2002.

SCHERER, M. J.; FEDERICI, S. Why people use and don't use technologies: Introduction to the special issue on assistive technologies for cognition/cognitive support technologies. NeuroRehabilitation, v. 37, n. 3, p. 315-319, 2015.

SCHERER, M. J.; GLUECKAUF, R. Assessing the benefits of assistive technologies for activities and participation. Rehabilitation Psychology, v. 50, n. 2, p. 132-141, 2005.

SCHERER, M. J.; et al. Predictors of assistive technology use: The importance of personal and psychosocial factors. Disability and Rehabilitation, v. 27, n. 21, p. 1321-1331, 2005.

SMITH, R. O. et al. Assistive technology products: a position paper from the first global research, innovation, and education on assistive technology (GREAT) summit. Disability and Rehabilitation: Assistive Technology, v. 13, n. 5, p. 473-485, 2018. 
VRKLJAN, B. Facilitating technology use in older adulthood: The person-environmentoccupation model revisited. British Journal of Occupational Therapy, v. 73, n. 9, p. 396404, 2010.

VROMAN, K. G.; ARTHANAT, S.; LYSACK, C. "Who over 65 is online?" Older adults' dispositions toward information communication technology. Computers in Human Behavior, v. 43, n. February, p. 156-166, 2015.

\section{Agradecimentos}

A FAPESP pelo auxílio financeiro para desenvolver essa pesquisa.

\section{Correspondência}

Ligia Maria Presumido Braccialll - Universidade Estadual Paulista. Av. Hygino Muzzi Filho, 737, Jardim Universitário. CEP: 17525900. Marília, São Paulo, Brasil.

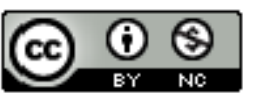

This work is licensed under a Creative Commons Attribution-NonCommercial 4.0 International (CC BY-NC 4.0) 